\title{
Increased prevalence of non-alcoholic fatty liver disease in European women with a history of gestational diabetes
}

\author{
S. Forbes • S. D. Taylor-Robinson • N. Patel • P. Allan • \\ B. R. Walker • D. G. Johnston
}

Received: 7 November 2010 / Accepted: 12 November 2010/Published online: 12 December 2010

(C) Springer-Verlag 2010

\begin{abstract}
Aims/hypothesis Non-alcoholic fatty liver disease (NAFLD) is common in type 2 diabetes but it is unknown whether NAFLD is prevalent in European women at risk of type 2 diabetes. We studied the prevalence of, and risk factors for, NAFLD in European women with previous gestational diabetes (GDM) at high risk of type 2 diabetes. Methods A total of 110 women with previous GDM and 113 without previous GDM, with non-diabetic glucose tolerance were recruited retrospectively from antenatal databases. Participants underwent liver ultrasound scan examination, anthropometry and blood sampling for liver function tests and to determine levels of fasting lipids, NEFA and insulin and glucose concentrations in order to derive insulin sensitivity and insulin secretion indices (HOMA\%S and HOMA\%B, respectively).

Results There was no significant difference in BMI in women with previous GDM compared with those without previous $\operatorname{GDM}\left(28.9 \pm 0.6\right.$ vs. $27.9 \pm 0.6 \mathrm{~kg} / \mathrm{m}^{2}$, respectively; $\left.p=0.12\right)$. Women with previous GDM had higher fasting and $2 \mathrm{~h}$
\end{abstract}

S. Forbes $(\bowtie) \cdot$ B. R. Walker

Endocrinology Unit, Centre for Cardiovascular Science,

Queen's Medical Research Institute, University of Edinburgh,

47 Little France Crescent,

Edinburgh EH16 4TJ, UK

e-mail: Shareen.Forbes@ed.ac.uk

S. D. Taylor-Robinson • N. Patel • D. G. Johnston

Division of Diabetes, Endocrinology and Metabolism,

Department of Medicine, Imperial College London,

London, UK

P. Allan

Department of Radiology, New Royal Infirmary Edinburgh,

Edinburgh, UK glucose concentrations following a 75 g OGTT ([mean \pm SEM] fasting glucose $5.3 \pm 0.1$ vs. $5.1 \pm 0.1 \mathrm{mmol} / 1, p=0.02$; $2 \mathrm{~h}$ glucose $6.8 \pm 0.2$ vs. $5.8 \pm 0.3 \mathrm{mmol} / \mathrm{l}, p=0.02$ ), dyslipidaemia (LDL-cholesterol $3.3 \pm 0.1$ vs. $2.8 \pm 0.1 \mathrm{mmol} / 1$; HDLcholesterol [median \{interquartile range\}] $1.3[1.2-1.6]$ vs. $1.8[1.5-1.9] \mathrm{mmol} / \mathrm{l}$; triacylglycerol $1.3[0.9-1.6]$ vs. 1.0 [0.7-1.7] mmol/l, all $p \leq 0.03$ ), higher insulin secretion and lower insulin sensitivity. NAFLD prevalence was greater in women with previous GDM compared with those without previous GDM: $38 \%(95 \%$ CI $28-47 \%)$ vs. $17 \%$ (95\% CI $10-24 \%), p=0.001$. In multiple logistic regression analysis, lower insulin sensitivity and raised serum alanine transaminase concentrations were associated with NAFLD.

Conclusions/interpretation NAFLD is prevalent in European women with previous GDM. Impaired insulin sensitivity and increased liver transaminase activity are closely associated with NAFLD in these women.

Keywords Alanine transaminase - Gestational diabetes . Insulin sensitivity · Non-alcoholic fatty liver disease · Type 2 diabetes

$\begin{array}{ll}\text { Abbreviations } \\ \text { ALT } & \text { Alanine transaminase } \\ \text { FFM } & \text { Fat-free mass } \\ \text { GDM } & \text { Gestational diabetes } \\ \gamma \mathrm{GT} & \text { Gamma-glutamyltransferase } \\ \text { IFG } & \text { Impaired fasting glucose } \\ \text { IGT } & \text { Impaired glucose tolerance } \\ \text { NAFLD } & \text { Non-alcoholic fatty liver disease } \\ \text { NGT } & \text { Normal glucose tolerance } \\ \text { TG } & \text { Triacylglycerol } \\ \text { US } & \text { Ultrasound }\end{array}$




\section{Introduction}

Non-alcoholic fatty liver disease (NAFLD) is characterised by the presence of fat within the liver in people who do not drink significant quantities of alcohol [1]; it is the most common liver abnormality in the western world, with a prevalence of approximately $20-30 \%$ [2]. Individuals with NAFLD are at increased risk of mortality compared with the general population, with particularly high rates of cardiovascular disease [2]. NAFLD represents a spectrum of disease ranging from simple steatosis to steato-hepatitis, cirrhosis and endstage liver disease. The increased intrahepatocellular triacylglycerol (TG) results when the rate of hepatic fatty acid uptake from plasma and de novo fatty acid synthesis is greater than the rate of fatty acid oxidation and export (as TG within VLDL) [3]. The presence of steatosis is associated with alterations in glucose, fatty acid and lipoprotein metabolism and NAFLD is strongly associated with type 2 diabetes and the metabolic syndrome [4]. Defects in insulin action and insulin secretion may be important in the pathogenesis of NAFLD and both increased $[4,5]$ and decreased [6] insulin secretion have been described in different populations. However, it is not clear whether NAFLD is present in all populations at risk of developing type 2 diabetes and, in turn, it is not known whether NAFLD causes metabolic dysfunction, including hyperglycaemia, or whether the metabolic dysfunction causes NAFLD.

Results from studies that address whether NAFLD is a risk factor for the development of type 2 diabetes in non-selected populations are inconsistent. Two longitudinal studies in Asian populations using ultrasound (US)-diagnosed NAFLD have demonstrated that NAFLD is a risk factor for the development of type 2 diabetes in Koreans [7] and Japanese men [8]. In contrast, a study in a Japanese cohort that included women [9] failed to show this relationship. Hence, the emergence of NAFLD prior to the development of frank diabetes is not well studied in women.

Gestational diabetes (GDM) is carbohydrate intolerance first diagnosed during pregnancy. Women with a history of GDM have a lifetime risk of type 2 diabetes in excess of $60 \%$ [10] and ethnicity-specific prevalence of GDM and type 2 diabetes are closely related [11].

In our present study we aimed to compare the prevalence of NAFLD in European women with a history of GDM with European women with no history of GDM. We also aimed to determine the variables most closely associated with NAFLD using logistic regression analysis.

\section{Methods}

Participants European women with and without previous GDM were recruited retrospectively via NHS hospital antenatal care databases. These databases record all maternity information and birth outcomes.

Tests in index pregnancy and diagnosis of GDM The presence or absence of GDM was ascertained from information in the clinical records. As part of routine clinical care, all obese participants (booking BMI $\geq 30 \mathrm{~kg} / \mathrm{m}^{2}$ ) and those with specific WHO risk factors for GDM (older age, previous history of large-for-gestational-age infant, raised fasting or random glucose) [12] had a $2 \mathrm{~h} 75 \mathrm{~g}$ OGTT at 24-28 weeks' gestation. Those participants who had no risk factors for GDM and who were not obese at antenatal booking $\left(\mathrm{BMI} \geq 20<30 \mathrm{~kg} / \mathrm{m}^{2}\right.$ ) had testing for glycosuria and a random plasma glucose measured.

Women were categorised as not having had GDM if they had either a normal $2 \mathrm{~h} 75 \mathrm{~g}$ OGTT by WHO criteria [12] or, if not obese, no glycosuria and a random venous plasma glucose $<9 \mathrm{mmol} / \mathrm{l}$ during the index or previous pregnancies where applicable. Women were classified as having had GDM using the results of a $2 \mathrm{~h} 75 \mathrm{~g}$ OGTT at 24-28 weeks' gestation and WHO criteria: fasting venous plasma glucose $\geq 7 \mathrm{mmol} / \mathrm{l}$ or $2 \mathrm{~h}$ venous plasma glucose $\geq 7.8 \mathrm{mmol} / \mathrm{l}$.

Selection of participants at recall European women with and without previous GDM who had had live births from 1 to 9 years previously were selected from the NHS antenatal care database and the women aged 20 years or older but under 45 years were further stratified into age groups of 5 year intervals. Equal numbers of women with and without previous GDM were invited to participate from these groups. This sampling was done as the prevalence of GDM and NAFLD increase with age and this would potentially be a source of bias in the study. As we wished to recruit participants who were not breastfeeding and who had non-diabetic glucose tolerance, we selected women who were more than 1 year but less than 10 years postpartum.

At postnatal recall, women with and without previous GDM had a 2 h 75 g OGTT and non-diabetic glucose tolerance according to WHO criteria: normal glucose tolerance (NGT) was defined as a fasting plasma glucose $<7 \mathrm{mmol} / \mathrm{l}$ and $2 \mathrm{~h}$ glucose $<7.8 \mathrm{mmol} / \mathrm{l}$; impaired fasting glucose (IFG) was defined as a fasting plasma glucose $\geq 6.1$, but $<7.0 \mathrm{mmol} / \mathrm{l}$; and impaired glucose tolerance (IGT) as a $2 \mathrm{~h}$ plasma glucose $\geq 7.8$, but $<11.1 \mathrm{mmol} / \mathrm{l}$, and a fasting plasma glucose $<7.0 \mathrm{mmol} / \mathrm{l}$. All women recruited were not pregnant or breastfeeding or on any medication that would affect metabolism (including insulin-sensitising agents, statins and anti-hypertensive medications) and had maintained a constant weight for the previous 12 months, as assessed by participant recall. All participants consumed $<20$ g alcohol per week, had a serum alanine transaminase (ALT) $<\times 2$ upper limit of normal (40 U/1), negative autoimmune antibodies (anti-nuclear antibody [titre $<40$ ], 
anti-mitochondrial, anti-smooth muscle and anti-liver kidney microsome antibodies), negative viral antibodies (hepatitis B surface antigen, hepatitis C antibody), normal ferritin levels and normal renal and thyroid function.

Participants provided written informed consent and the study was approved by institutional review boards (St Mary's Hospital Ethics Committee and Lothian Health Ethics Committee), and was conducted in accordance with the principles endorsed by the Declaration of Helsinki.

Seven women without previous GDM and six women with previous GDM were identified with positive autoantibodies and abnormal liver function; they were referred to a hepatologist for further investigation and management and excluded from the study. Therefore, 110 women with previous GDM and 113 women without previous GDM were eligible for recruitment into the study and underwent the following investigations.

Diagnosis of hepatic steatosis The presence of intrahepatic fat was assessed by US scan following an $8-10 \mathrm{~h}$ fast using a Siemens ACUSON system with a $3.5 \mathrm{MHz}$ transducer (Siemens Medical Systems, Issaquah, WA, USA). Detection of hepatic fat was based on an echogenic parenchyma (in relation to the right kidney), posterior attenuation and the presence of focal fatty sparing [13]. Two sonographers performed all of the US scans. The stored images of all participants were assessed at the end of the study by an independent consultant radiologist blinded to the clinical history. Participants were given an overall liver grading based on a subjective measurement of the severity of steatosis: normal (i.e. no or a possible slight increase in echogenicity or slightly impaired visualisation of the diaphragm or intrahepatic vessels); mild steatosis (i.e. definite increase in echogenicity and/or definite impaired visualisation of the intrahepatic vessels and diaphragm, with or without evidence of focal fatty sparing); severe steatosis (i.e. marked increase in echogenicity and/or poor or no visualisation of the diaphragm and intrahepatic vessels, with or without focal fatty sparing).

Other measurements The participants attended the clinical research facilities at 08:00 hours following an overnight (8-12 h) fast. Body weight was determined on an electronic scale. Fat mass and fat-free mass (FFM) were measured by bioelectrical impedance analysis (TBF-300M; TANITA Corporation, Tokyo, Japan). Waist and hip circumference measurements were made to the nearest $0.5 \mathrm{~cm}$, with the participants standing, halfway between the spina iliaca and the last rib, and at the trochanter major, respectively. Blood pressure was recorded by a semi-automatic digital sphygmomanometer (Omron 705CP blood pressure monitor; Omron Healthcare, Milton Keynes, UK) to the nearest $\mathrm{mmHg}$.
Blood samples were taken at this visit for liver function tests, autoimmune and viral antibodies, ferritin, thyroid and renal function tests, NEFA level and lipid profile. Two blood samples were taken $>10 \mathrm{~min}$ apart to determine fasting insulin and glucose levels.

Biochemical analyses Glucose concentrations were measured by a hexokinase method (Randox Laboratories, Crumlin, Co. Antrim, UK) on a microplate reader (OPTImax; Molecular Devices, Wokingham, UK). NEFA concentrations were measured by a colorimetric method (Wako Chemicals, Neuss, Germany) on a clinical analyser (Cobas Fara, Hoffman-La Roche, Basel, Switzerland). Serum samples for insulin were analysed in duplicate by an ELISA (Mercodia, Uppsala, Sweden) on a microplate reader (Thermomax; Molecular Devices). All the metabolic analyses were done together at the end of the study.

Assessment of insulin secretion and insulin action HOMA (HOMA2 index; available from www.dtu.ox.ac.uk, accessed 9 September 2010) was performed on the mean of two fasting glucose and two fasting insulin concentrations to provide an analysis of insulin secretion (HOMA $\% \mathrm{~B})$ and insulin sensitivity $(\mathrm{HOMA} \% \mathrm{~S})$ in these patients.

Statistical power and analysis Based on previous literature, we estimated that our women without previous GDM would have a prevalence of NAFLD of $20 \%$ [2]. A sample size of $n=100$ per group has $90 \%$ power to detect a $15 \%$ difference in prevalence between the groups with and without previous GDM.

Data are presented as the mean $\pm \mathrm{SEM}$ or, in the case of skewed data, the median and interquartile ranges. Statistical analyses were performed in STATA 8 (Stata Corporation, College Station, TX, USA). Univariate analyses comparing the women with and without a previous history of GDM used the unpaired Student's $t$ test and Mann-Whitney $U$ test, as appropriate. The groups were combined and univariate logistic regression was performed on BMI. Logistic regression analysis was performed on selected variables, each adjusted for BMI, to determine their relationship with NAFLD. Multiple logistic regression analysis was performed with a number of variables to establish which of these were independently associated with NAFLD. A $p$ value $<0.05$ was taken as significant.

\section{Results}

Participant characteristics during index pregnancy During pregnancy, 95 women with GDM were diet controlled and 15 women required insulin treatment. Amongst women 
with GDM, this was the first pregnancy in 67 participants, the second pregnancy in 37 participants and the third pregnancy in six participants that was complicated by GDM. There was no significant difference between women with and without GDM in age $(33 \pm 1$ vs. $33 \pm 1$ years, respectively, $p=0.7$ ), parity ( $3 \pm 0$ vs. $2 \pm 0, p=0.14$ ), or BMI at term $\left(27.8 \pm 0.6\right.$ vs. $\left.26.8 \pm 0.7 \mathrm{~kg} / \mathrm{m}^{2}, p=0.23\right)$.

Participant characteristics at recall Women with previous GDM vs. those without previous GDM were studied $6 \pm 0$ vs. $7 \pm 0$ years following their index pregnancy. Participant characteristics are shown in Table 1. Participants were predominantly overweight. Women with previous GDM had non-significantly greater BMI than those without previous GDM. There was no significant difference in age between the groups. Women with previous GDM had higher waist circumference, fat mass and diastolic blood pressure.

The metabolic data are shown in Table 2. The majority of women in both groups had normal glucose tolerance: $82 \%$ vs. $88 \%$ in women with previous GDM vs. those without previous GDM. The distribution of glucose tolerance in the two groups differed with the majority of hyperglycaemic women with previous GDM in the IFG group and the majority of hyperglycaemic women without previous GDM in the IGT group $\left(\chi^{2}=8.01,3 d f, p=0.04\right)$. Women with previous GDM had higher fasting insulin concentrations, were less insulin sensitive (HOMA\%S) and had greater insulin secretion (HOMA\%B) than women without previous GDM. Circulating TG and LDL-cholesterol concentrations were raised and HDL-cholesterol concentrations diminished in the women with previous GDM. There were no differences in NEFA concentrations, serum ALT and gammaglutamyltransferase $(\gamma \mathrm{GT})$ between the groups.

The prevalence of NAFLD was significantly greater in the women with previous GDM vs. women without previous GDM: $38 \%(95 \%$ CI $28-47 \%)$ vs. $17 \%$ (95\% CI
$10-24 \%), p=0.001$. The OR of NAFLD in the women with previous GDM, adjusted for BMI, was more than double that in the women with no previous GDM (OR 2.77 [95\% CI 1.43-5.37], $p=0.002$ ).

The distribution of women with previous GDM vs. those without previous GDM into the three US-scan-graded categories of normal, mild and severe hepatic steatosis was significantly different $\left(\chi^{2}=13.6,2 d f, p=0.001\right.$; Table 3$)$, with no cases of severe hepatic steatosis in the women without previous GDM.

Although hyperglycaemia occurred in only $18 \%$ and $12 \%$ of women with and without previous GDM, respectively, cases of NAFLD occurred disproportionately more frequently in the hyperglycaemic groups: $38 \%$ and $58 \%$ of NAFLD cases were in the hyperglycaemic groups in women with and without previous GDM, respectively $\left(\chi^{2}=18,1 d f\right.$ and $\chi^{2}=40,1 d f$, respectively, both $p<$ 0.0001; Table 4). In those women with NAFLD, ALT concentrations were greatest in the hyperglycaemic subgroups vs. the normoglycaemic group (Table 4).

On univariate logistic regression, BMI was strongly associated with NAFLD (Table 5). In view of the nonsignificantly greater BMI in women with previous GDM, logistic regression analyses were adjusted for BMI. On bivariate logistic regression, correcting individual variables for BMI only, a history of previous GDM was strongly associated with NAFLD but notably insulin secretion (HOMA\%B) and NEFA concentrations were not statistically associated with NAFLD (Table 5). In multiple logistic regression analysis, only diminished insulin sensitivity (HOMA\%S) (OR 0.08 [95\% CI 0.04-0.19] per $1 \mathrm{SD}, p<$ 0.001 ) and raised ALT concentrations (OR 2.85 [95\% CI 1.63-4.98] per $1 \mathrm{SD}, p<0.001)$ were independently associated with NAFLD from the variables: group (i.e. previous GDM vs. those without previous GDM); insulin sensitivity (HOMA\%S); ALT; fasting TG concentrations; and BMI.
Table 1 Characteristics of participants

Data are mean \pm SEM in European women with previous GDM vs. women with no previous GDM

Groups were compared by univariate analyses using the unpaired Student's $t$ test or Mann-Whitney $U$ test, as appropriate

$\mathrm{BP}$, blood pressure

\begin{tabular}{llll}
\hline Characteristic & Previous GDM & No previous GDM & $p$ value \\
\hline$n$ & 110 & 113 & - \\
Age (years) & $39 \pm 1$ & $39 \pm 1$ & 0.61 \\
Height $(\mathrm{m})$ & $1.63 \pm 0.01$ & $1.64 \pm 0.01$ & 0.57 \\
Weight $(\mathrm{kg})$ & $76.7 \pm 1.6$ & $73.7 \pm 1.32$ & 0.2 \\
BMI $\left(\mathrm{kg} / \mathrm{m}^{2}\right)$ & $28.9 \pm 0.6$ & $27.9 \pm 0.6$ & 0.12 \\
Systolic BP (mmHg) & $124 \pm 2$ & $120 \pm 1$ & 0.08 \\
Diastolic BP (mmHg) & $75 \pm 1$ & $72 \pm 1$ & 0.03 \\
Waist circumference $(\mathrm{cm})$ & $89 \pm 1$ & $84 \pm 1$ & 0.002 \\
Hip circumference $(\mathrm{cm})$ & $107 \pm 1$ & $105 \pm 1$ & 0.29 \\
Fat mass $(\mathrm{kg})$ & $29.7 \pm 1.2$ & $26.9 \pm 0.70$ & 0.001 \\
FFM (kg) & $46.9 \pm 0.6$ & $46.3 \pm 0.4$ & 0.40 \\
\hline
\end{tabular}


Table 2 Metabolic data in European women with previous GDM compared with women with no previous GDM

\begin{tabular}{|c|c|c|c|}
\hline Metabolic variable & Previous GDM & No previous GDM & $p$ value \\
\hline Fasting plasma glucose $(\mathrm{mmol} / \mathrm{l})$ & $5.3 \pm 0.1$ & $5.1 \pm 0.1$ & 0.02 \\
\hline $2 \mathrm{~h}$ plasma glucose $(\mathrm{mmol} / \mathrm{l})$ & $6.8 \pm 0.2$ & $5.8 \pm 0.3$ & 0.02 \\
\hline NGT $(\%)$ & 82 & 88 & $*$ \\
\hline IFG $(\%)$ & 18 & 6 & $*$ \\
\hline IGT $(\%)$ & 6 & 11 & $*$ \\
\hline IFG $(\%)+$ IGT $(\%)$ & 6 & 4 & $*$ \\
\hline Fasting insulin (pmol/1) & $57(40-114)$ & $34(24-49)$ & $<0.001$ \\
\hline НОМА $\%$ В & $97(79-126)$ & $64(61-81)$ & $<0.001$ \\
\hline HOMA $\% \mathrm{~S}$ & $89(47-137)$ & $154(103-228)$ & $<0.001$ \\
\hline Plasma ALT (U/1) (NR 10-50) & $27(15-30)$ & $21(16-28)$ & 0.41 \\
\hline Plasma $\gamma \mathrm{GT}(\mathrm{U} / \mathrm{l})(\mathrm{NR}$ 5-35) & $19(11-27)$ & $17(12-29)$ & 0.61 \\
\hline Fasting plasma TG (mmol/1) (NR 0.8-2.1) & $1.3(0.9-1.6)$ & $1.0(0.7-1.7)$ & 0.03 \\
\hline Fasting plasma cholesterol (mmol/l) & $5.3 \pm 0.1$ & $5.2 \pm 0.1$ & 0.88 \\
\hline Fasting plasma HDL-cholesterol (mmol/l) & $1.3(1.2-1.6)$ & $1.8(1.5-1.9)$ & $<0.001$ \\
\hline Fasting plasma LDL-cholesterol (mmol/l) & $3.3 \pm 0.1$ & $2.8 \pm 0.1$ & 0.001 \\
\hline Fasting plasma NEFA $(\mu \mathrm{mol} / \mathrm{l})$ & $666 \pm 19$ & $649 \pm 13$ & 0.49 \\
\hline
\end{tabular}

Data are mean \pm SEM or median (interquartile range) in the case of skewed data

For conversion from SI units in the table to conventional units: multiply by 18.018 to convert glucose to mg/dl; divide by 6.945 to convert insulin to $\mu \mathrm{U} / \mathrm{ml}$; multiply by 38.610 to convert cholesterol to $\mathrm{mg} / \mathrm{dl}$; multiply by 88.496 to convert $\mathrm{TG}$ to $\mathrm{mg} / \mathrm{dl}$

Groups were compared by univariate analyses using the unpaired Student's $t$ test or Mann-Whitney $U$ test, as appropriate; distributions in glucose tolerance were compared between groups using Pearson's $\chi^{2}$ test, ${ }^{*} p=0.04$

NR, normal range

\section{Discussion}

Our study shows a significantly greater prevalence of NAFLD in non-diabetic women with a history of GDM compared with women with no previous history of GDM. The women with previous GDM were studied $<10$ years after their index pregnancy and had non-diabetic glucose tolerance. Prospective studies have estimated that these women are at $~ 60 \%$ risk of future diabetes 10 to 16 years after their index pregnancy [14]. Therefore, NAFLD is prevalent in these women at risk for the development of type 2 diabetes.

On univariate logistic regression analysis, BMI was significantly associated with NAFLD. It is well established that the prevalence of NAFLD increases with increasing BMI [1]. As BMI increases, so too does fat mass and the potential for NEFA delivery to the liver from different fat depots increases. After adjusting for BMI, the OR for NAFLD in the women with previous GDM was more than double that in women without previous GDM. In multiple logistic regression analysis, including the variables group (i.e. women with previous GDM vs. those with no previous GDM), insulin sensitivity (HOMA\%S), ALT, fasting TG concentrations and BMI, a history of previous GDM was not independently associated with NAFLD. However, multiple logistic regression analysis in this context has its limitations as many of the components in the model represent a cluster of metabolic defects associated with a history of previous GDM and these metabolic variables are inter-related [15]. As such, the relationship between previous GDM and NAFLD may be attenuated to the null in this model secondary to an over adjustment by these other metabolic variables.

Table 3 US scan grades of steatosis

\begin{tabular}{llll}
\hline Group & No steatosis & Mild steatosis & Severe steatosis \\
\hline Previous GDM, \% $(n)$ & $62(68)$ & $37(40)$ & $2(2)$ \\
No previous GDM, \% $(n)$ & $83(94)$ & $17(19)$ & $0(0)$ \\
\hline
\end{tabular}

Participants with previous GDM and no previous GDM and the different grades of steatosis: normal/no steatosis, mild steatosis and severe steatosis, as determined by US scan

The difference in the distribution of the women with and without previous GDM in these groups was compared using Pearson's $\chi^{2}$ test, $p=0.001$ 
Table 4 Prevalence of NAFLD and ALT concentrations according to glycaemic status in women with and without previous GDM

\begin{tabular}{lllll}
\hline Group & \multicolumn{2}{l}{ Previous GDM } & & \multicolumn{2}{l}{ No previous GDM } \\
\cline { 2 - 3 } & NAFLD, \% $(n)$ & ALT & NAFLD, \% $(n)$ & ALT \\
\hline NGT & $62(26)$ & $30(28-41)$ & $32(8)$ & $24(15-28)$ \\
IFG & $38(16)$ & $32(24-43)$ & $47(6)$ & $29(18-36)$ \\
IGT & $12(5)$ & $34(32-47)$ & $21(4)$ & $29(26-36)$ \\
IFG+IGT & $12(5)$ & $34(32-47)$ & $<0.0001^{\mathrm{a}}$ & $0.03^{\mathrm{b}}$ \\
$p$ value & $<0.0001^{\mathrm{a}}$ & $0.02^{\mathrm{b}}$ & & \\
\hline
\end{tabular}

Prevalence of NAFLD according to glucose tolerance with corresponding ALT concentrations (median [interquartile range])

${ }^{a}$ Pearson's $\chi^{2}$ tests were performed with NGT/'hyperglycaemia' vs. presence/absence of NAFLD in each group

${ }^{\mathrm{b}}$ Mann-Whitney $U$ tests were performed comparing ALT concentrations in each group with NGT vs. those with hyperglycaemia

Cases of NAFLD occurred disproportionately more frequently in the hyperglycaemic subgroups, consistent with previous studies in other populations [4]. Circulating ALT concentrations were greatest in those participants with NAFLD associated with hyperglycaemia and in the multiple logistic regression analysis, ALT was significantly and independently associated with NAFLD. Rather than simply being a marker of hepatocellular damage, ALT may play a role in the pathogenesis of type 2 diabetes. ALT catalyses the reversible transamination between L-alanine and $\alpha$-ketoglutarate to form pyruvate and L-glutamate, influencing gluconeogenesis and amino acid metabolism

Table 5 Logistic regression for the presence of NAFLD

\begin{tabular}{lllll}
\hline Variable & $1 \mathrm{SD}$ & OR & $95 \%$ CI & $p$ value \\
\hline BMI (kg) & 5 & 1.89 & $1.38-2.59$ & $<0.001$ \\
GDM & - & 2.77 & $1.43-5.37$ & 0.002 \\
Fasting insulin (pmol/1) & 53 & 3.67 & $2.24-6.02$ & $<0.001$ \\
HOMA\%B (\%) & 41 & 1.34 & $0.99-1.91$ & 0.06 \\
HOMA\%S (\%) & 77 & 0.12 & $0.06-0.23$ & $<0.001$ \\
ALT (U/l) & 13 & 1.42 & $1.05-1.93$ & 0.02 \\
Fasting glucose (mmol/l) & 0.7 & 2.66 & $1.83-3.88$ & $<0.001$ \\
NEFA (mmol/l) & 0.21 & 1.12 & $0.78-1.58$ & 0.57 \\
TG (mmol/l) & 0.56 & 1.89 & $1.37-2.63$ & $<0.001$ \\
\hline
\end{tabular}

The groups were combined and the potential explanatory variables were adjusted for BMI and explored in a bivariate logistic regression model

For conversion from SI units in the table to conventional units: divide by 6.945 to convert insulin to $\mu \mathrm{U} / \mathrm{ml}$; multiply by 18.018 to convert glucose to $\mathrm{mg} / \mathrm{dl}$; multiply by 88.496 to convert TG to $\mathrm{mg} / \mathrm{dl}$

The OR is defined per SD of the continuous variable: an $\mathrm{OR}>1.00$ with $p<0.05$ implies the explanatory variable adjusted for BMI is a risk factor for NAFLD; an OR $<1.00$ with $p<0.05$ implies the explanatory variable adjusted for BMI is a protective factor for NAFLD

HOMA $\%$ S, insulin sensitivity index; $\mathrm{HOMA} \% \mathrm{~B}$, insulin secretion index in the liver, adipose tissue and other organs. Longitudinal studies have mainly examined male cohorts and some have shown that abnormalities in ALT may precede the development of type 2 diabetes [16, 17]. Few studies have examined female cohorts alone, but in some that have, ALT has not appeared to be predictive of type 2 diabetes [18, 19]. However, a recent meta-analysis of population-based prospective studies concluded that ALT was associated with a future risk of type 2 diabetes [20]. The mechanism of this association has remained uncertain, because few of these studies included US diagnosis of NAFLD, and not all studies excluded other causes of liver disease. In the National Health and Nutrition Examination Survey, 31\% of the elevated aminotransferase activity could be explained by high alcohol consumption and/or hepatitis $\mathrm{B}$ or $\mathrm{C}$ viral infection [21]. In our study, women were carefully selected to have an alcohol intake $<20 \mathrm{~g} / \mathrm{week}$, had negative viral and autoimmune serology and were not on hepatotoxic medication. Therefore, an elevated ALT may be linked with liver fat accumulation and future type 2 diabetes in these European women; long-term prospective clinical studies are required to confirm this.

The women with previous GDM had diminished insulin sensitivity (HOMA\%S), increased insulin secretion (HOMA\%B) and were hyperinsulinaemic compared with women with no previous history of GDM. In multiple logistic regression analysis, diminished insulin sensitivity was independently associated with NAFLD in this population in common with other populations $[4,5]$. Impaired insulin sensitivity would lead to diminished suppression of hepatic glucose production and diminished suppression of NEFA from adipose tissue [22], leading to an increased flux of NEFA to the liver which may lead to hepatic steatosis. In contrast, neither impaired nor enhanced insulin secretion was associated with NAFLD when adjusted for BMI in these women with previous GDM. This result is consistent with a recent cross-sectional study in obese children, which used the ratios of the integrated area under the curves of 
insulin to glucose following a $2 \mathrm{~h} 75 \mathrm{~g}$ OGTT as an index of insulin secretion [23]. However, some smaller mechanistic studies have shown an association with enhanced insulin secretion and NAFLD [4,5] and this warrants further study in this population of women using more discriminatory methods to study insulin secretion.

Hyperinsulinaemia was an important variable associated with NAFLD. High fasting insulin concentrations may inhibit VLDL-TG secretion from the liver and lead to steatosis.

The main limitation of our study is the cross-sectional design and so a causal relationship between NAFLD and the development of type 2 diabetes cannot be inferred. The other limitation is that not all of the women with a history of previous GDM will go on to develop type 2 diabetes. Therefore, further prospective studies are required in this cohort of women.

In conclusion, we have demonstrated for the first time that US-diagnosed NAFLD is significantly more prevalent in European women with a history of GDM compared with European women with no history of GDM. As these women are at significant risk of type 2 diabetes, this result may be consistent with NAFLD preceding type 2 diabetes in this cohort. Diminished insulin sensitivity and raised ALT activity may all contribute to the development of NAFLD in these women.

Acknowledgements S. Forbes was supported by a Diabetes UK Clinical Intermediate Fellowship (BDA:RD04/0002483). S. D. TaylorRobinson, N. Patel and D. G. Johnston are grateful to the NIHR Biomedical Facility at Imperial College, London, for infrastructure support. N. Patel was funded by a grant from the British Medical Research Council to S. D. Taylor-Robinson (G99000178). We acknowledge the support of the British Heart Foundation Centre of Research Excellence. The research was conducted in the Metabolic Day Ward (St Mary's Hospital, London, UK) and the Clinical Research Facility (Royal Infirmary, Edinburgh, UK). We thank C. Graham and G. McHugh for statistical advice and are particularly grateful to the women who participated.

Duality of interest The authors declare that there is no duality of interest associated with this manuscript.

\section{References}

1. Angulo P (2002) Nonalcoholic fatty liver disease. N Engl J Med 346:1221-1231

2. Targher G, Bertolini L, Padovani R et al (2007) Prevalence of nonalcoholic fatty liver disease and its association with cardiovascular disease among type 2 diabetic patients. Diabetes Care 30:1212-1218

3. Postic C, Girard J (2008) Contribution of de novo fatty acid synthesis to hepatic steatosis and insulin resistance: lessons from genetically engineered mice. J Clin Invest 118:829-838

4. Pagano G, Pacini G, Musso G et al (2002) Nonalcoholic steatohepatitis, insulin resistance, and metabolic syndrome: further evidence for an etiologic association. Hepatology 35:367-372
5. Chitturi S, Abeygunasekera S, Farrell GC et al (2002) NASH and insulin resistance: insulin hypersecretion and specific association with the insulin resistance syndrome. Hepatology 35:373-379

6. Musso G, Gambino R, Biroli G et al (2005) Hypoadiponectinemia predicts the severity of hepatic fibrosis and pancreatic beta-cell dysfunction in nondiabetic nonobese patients with nonalcoholic steatohepatitis. Am J Gastroenterol 100:2438-2446

7. Kim CH, Park JY, Lee KU, Kim JH, Kim HK (2008) Fatty liver is an independent risk factor for the development of type 2 diabetes in Korean adults. Diabet Med 25:476-481

8. Shibata M, Kihara Y, Taguchi M, Tashiro M, Otsuki M (2007) Nonalcoholic fatty liver disease is a risk factor for type 2 diabetes in middle-aged Japanese men. Diabetes Care 30:2940-2944

9. Okamoto M, Takeda Y, Yoda Y, Kobayashi K, Fujino MA, Yamagata $Z$ (2003) The association of fatty liver and diabetes risk. J Epidemiol 13:15-21

10. O'Sullivan JB (1991) Diabetes mellitus after GDM. Diabetes 40 (Suppl 2):131-135

11. Yue DK, Molyneaux LM, Ross GP, Constantino MI, Child AG, Turtle JR (1996) Why does ethnicity affect prevalence of gestational diabetes? The underwater volcano theory. Diabet Med 13:748-752

12. WHO (1999) Definition, diagnosis and classification of diabetes mellitus and its complications. Report of a WHO consultation. WHO, Geneva

13. Sanyal AJ (2002) AGA technical review on nonalcoholic fatty liver disease. Gastroenterology 123:1705-1725

14. O'Sullivan JB (1982) Body weight and subsequent diabetes mellitus. JAMA 248:949-952

15. Dornhorst A, Bailey PC, Anyaoku V, Elkeles RS, Johnston DG, Beard RW (1990) Abnormalities of glucose tolerance following gestational diabetes. Q J Med 77:1219-1228

16. Sattar N, Scherbakova O, Ford I et al (2004) Elevated alanine aminotransferase predicts new-onset type 2 diabetes independently of classical risk factors, metabolic syndrome, and C-reactive protein in the west of Scotland coronary prevention study. Diabetes 53:2855-2860

17. Ohlson LO, Larsson B, Bjorntorp P et al (1988) Risk factors for type 2 (non-insulin-dependent) diabetes mellitus. Thirteen and one-half years of follow-up of the participants in a study of Swedish men born in 1913. Diabetologia 31:798-805

18. Andre P, Balkau B, Born C et al (2005) Hepatic markers and development of type 2 diabetes in middle aged men and women: a three-year follow-up study. The D.E.S.I.R. Study (Data from an Epidemiological Study on the Insulin Resistance syndrome). Diabetes Metab 31:542-550

19. Nannipieri M, Gonzales C, Baldi S et al (2005) Liver enzymes, the metabolic syndrome, and incident diabetes: the Mexico City diabetes study. Diabetes Care 28:1757-1762

20. Fraser A, Harris R, Sattar N, Ebrahim S, Davey Smith G, Lawlor DA (2009) Alanine aminotransferase, gammaglutamyltransferase, and incident diabetes: the British Women's Heart and Health Study and meta-analysis. Diabetes Care 32:741-750

21. Clark JM, Brancati FL, Diehl AM (2003) The prevalence and etiology of elevated aminotransferase levels in the United States. Am J Gastroenterol 98:960-967

22. Forbes S, Robinson S, Dungu J et al (2006) Sustained endogenous glucose production, diminished lipolysis and non-esterified fatty acid appearance and oxidation in non-obese women at high risk of type 2 diabetes. Eur J Endocrinol 155:469-476

23. Bedogni G, Gastaldelli A, Manco M et al (2010) Relationship between fatty liver and glucose metabolism: a cross-sectional study in 571 obese children. Nutr Metab Cardiovasc. doi:10.1016/ j.numecd.2010.05.003 